\title{
Black Hole Cosmology: A Biological Boom
}

\section{UVS Seshavatharam ${ }^{1 *}$ and S Lakshminarayana ${ }^{2}$}

${ }^{1}$ Honorary faculty, I-SERVE, Alakapuri, Hyderabad-35, AP, India

${ }^{2}$ Dept. of Nuclear Physics, Andhra University, Visakhapatnam-03, AP, India

\begin{abstract}
In the previously published papers the authors made an attempt to develop a possible model of Black hole cosmology in a constructive way. In this model, forever rotating at light speed, high temperature and high angular velocity small sized primordial cosmic black hole of mass $M_{C} \cong \sqrt{e^{2} / 4 \pi \varepsilon_{0} G}$ gradually transforms into a low temperature and low angular velocity large sized massive primordial cosmic black hole. At any time represents the angular velocity. At its ending stage of expansion, for the whole cosmic black hole as practically remains constant, its corresponding thermal energy density will be 'the same' throughout its volume. This 'sameness' may be the reason for the observed 'isotropic' nature of the current CMB radiation. Observed cosmic red shift can be re-interpreted as an index of cosmological galactic atomic light emission phenomenon. Validity of this model can be well confirmed from the combined study of cosmological and microscopic physical phenomena. Finally it can be suggested that, the cosmological time is real and absolute. By interconnecting the present cosmic time with the present CMBR energy density the authors made an attempt to fit and estimate the current cosmic age and its obtained magnitude is close to 282 trillion years.
\end{abstract}

Keywords: Black hole cosmology; CMBR energy density; Fine structure ratio; Cosmic Redshift; Cosmological discrete light emission mechanism; Cosmic time

\section{Introduction}

In our daily life generally it is observed that any animal or fruit or human beings (from birth to death) grows with closed boundaries (irregular shapes also can have a closed boundary). An apple grows like an apple. An elephant grows like an elephant. A plant grows like a plant. A human being grows like a human being. Throughout their life time they won't change their respective identities. These are observed facts. From these observed facts it can be suggested that "growth" or "expansion" can be possible with a closed boundary. Thinking that nature loves symmetry, in a heuristic approach in this paper authors assume that "throughout its life time universe is a primordial black hole". Even though it is growing, at any time it is having an event horizon with a closed boundary and thus it retains her identity as a black hole forever. Note that universe is an independent body. It may have its own set of laws. At any time if universe maintains a closed boundary to have its size minimum at that time it must follow "strong gravity" at that time. If universe is having no black hole structure - any massive body (which is bound to the universe) may not show a black hole structure. That is black hole structure may be a subset of cosmic structure. This idea may be given a chance.

In this paper by highlighting the following 12 major short comings of modern big bang cosmology the authors made an attempt to develop a possible model of Black hole cosmology in a constructive way [1-3]. From now onwards instead of focusing on 'big bang cosmology' it is better to concentrate on 'black hole cosmology'. Its validity can be well confirmed from a combined study of cosmological and microscopic physical phenomena. It can be suggested that, there exists one variable physical quantity in the presently believed atomic and nuclear physical constants and "rate of change" in its magnitude can be considered as a "standard measure" of the present "cosmic rate of expansion" [4].

According to Tinaxi Zhang [5-7], the universe originated from a hot star-like black hole with several solar masses and gradually grew up through a super massive black hole with billion solar masses to the present state with hundred billion-trillion solar masses by accreting ambient materials and merging with other black holes.
According to Poplawski [8-11], the Universe is the interior of an Einstein-Rosen black hole and began with the formation of the black hole from a supernova explosion in the center of a galaxy. He theorizes that torsion manifests itself as a repulsive force which causes fermions to be spatially extended and prevents the formation of a gravitational singularity within the black hole's event horizon. Because of torsion, the collapsing matter on the other side of the horizon reaches an enormous but finite density, explodes and rebounds, forming an Einstein-Rosen bridge (wormhole) to a new, closed, expanding universe. Analogously, the Big Bang is replaced by the Big Bounce before which the Universe was the interior of a black hole. The rotation of a black hole would influence the space-time on the other side of its event horizon and results in a preferred direction in the new universe.

Most recently cosmologists Razieh Pourhasan, Niayesh Afshordi and Robert B. Manna have proposed [12] that the Universe formed from the debris ejected when a four-dimensional star collapsed into a black hole - a scenario that would help to explain why the cosmos seems to be so uniform in all directions.

\section{Major Shortcomings of Modern Big Bang Cosmology}

A) The standard Big Bang model tells us that the Universe exploded out of an infinitely dense point, or singularity. But nobody knows what would have triggered this outburst: the known laws of physics cannot tell us what happened at that moment.

B) Really if there was a 'big bang' in the past, with reference to formation of the big bang as predicted by GTR and with reference to the cosmic expansion that takes place simultaneously in all

*Corresponding author: UVS Seshavatharam, Honorary faculty, I-SERVE, Alakapuri, Hyderabad, 500035, AP, India, Tel: 08106085844, E-mail: seshavatharam.uvs@gmail.com

Received January 20, 2014; Accepted February 06, 2014; Published February 08, 2014

Citation: Seshavatharam UVS, Lakshminarayana S (2014) Black Hole Cosmology: A Biological Boom. Astrobiol Outreach 2:108. doi: 10.4172/2332-2519.1000108

Copyright: () 2014 Seshavatharam UVS, et al. This is an open-access article distributed under the terms of the Creative Commons Attribution License, which permits unrestricted use, distribution, and reproduction in any medium, provided the original author and source are credited. 
directions at a uniform rate at that time about the point of big bang - 'point' of big bang can be considered as the centre or characteristic reference point of cosmic expansion in all directions. In this case, saying that there is no preferred direction in the expanding universe - may not be correct.

C) There is no scientific evidence for the Friedmann's second assumption. We believe it only on the grounds of modesty [13].

D) Dimensionally it is perfectly possible to show that, the dimensions of Hubble's constant and angular velocity are same. If so considering Hubble's constant merely as an expansion parameter may not be correct. Even though it was having strong footing, Mach's principle [14] was not implemented successfully. At any given cosmic time, the product of 'critical density' and 'Hubble volume' gives a characteristic cosmic mass and it can be called as the 'Hubble mass'. Interesting thing is that, Schwarzschild radius of the 'Hubble mass' again matches with the 'Hubble length'. Most of the cosmologists believe that this is merely a coincidence. Here the authors emphasize the fact that this coincidence is having deep connection with cosmic geometry and the cosmological physical phenomena.

E) It may be noted that, increased redshifts and increased distances forced Edwin Hubble to propose the Hubble's law $[15,16]$. In fact there is no chance or scope or place for 'galaxy receding'. It is only our belief in its 'given' (Doppler shift based) interpretation. Even then, merely by estimating galaxy distance and without measuring galaxy receding speed, one cannot verify its acceleration. Clearly speaking: two mistakes are possible here. 1) Assumed galaxy receding speed is not being measured and not being confirmed. 2) Without measuring and confirming the galaxy receding speed, how can one say and confirm that it (galaxy) is accelerating. It is really speculative and unfortunate also.

F) During cosmic expansion, assuming past and present galaxies (which actually found to have gigantic structures) as 'points' and guessing photons coming from that galactic point particles seem to be ad-hoc. If light is coming from the atoms of the gigantic galaxy, then redshift can be interpreted as an index of the galactic atomic 'light emission mechanism'. In no way it seems to be connected with 'galaxy receding'.

G) If cosmic expansion is continuous and accelerating and redshift is a measure of cosmic expansion, then 'rate of increase in redshift' can be considered as a measure of cosmic 'rate of expansion'. Then there is no possibility to observe a 'constant' red shift. More over the current definition of red shift seems to be ad-hoc and not absolute. Please see section 6 . Hence one may not be able to understand or confirm the actual cosmic rate of expansion.

$\mathrm{H})$ According to the modern cosmological approach, bound systems like 'atoms' which are found to be the major constituents of galactic matter - will not expand with cosmic expansion/ acceleration. As per the present observational data this may be true. It might be the result of ending stage of expansion also. In this regard, without considering and without analysing the past data, one cannot come to a conclusion. If it is not possible to collect the past data, theoretically it may be possible to proceed further in this new direction.

I) Even though the whole physics strictly follows the 'constancy of speed of light', cosmic acceleration seems to violate it. This is really doubtful.

J) Drop in 'cosmic temperature' can be considered as a measure of cosmic expansion and 'rate of decrease in cosmic temperature' can be considered as a measure of cosmic 'rate of expansion'. But if rate of decrease in temperature is very small and is beyond the scope of current experimental verification, then the two possible states are: a) cosmic temperature is decreasing at a very slow rate and universe is expanding at a very slow rate and b) there is no 'observable' thermal expansion and there is no 'observable' cosmic expansion.

K) If 'Dark energy' is the major outcome of the 'accelerating universe', it is very important to note that - in understanding the basic concepts of unification or other fundamental areas of physics, role of dark energy is very insignificant. So far no ground based experiment confirmed the existence of dark energy. There is no single clue or definition or evidence to any of the natural physical properties of (the assumed) dark energy.

Based on these short comings the concepts of 'big bang cosmology' can be relinquished. The subject of cosmology is open. If one is willing to think in this new direction, certainly other hidden things can also be surfaced out. Most of the modern cosmologists are enforced with 85 years old Hubble's interpretation. This is the time to re-interpret the Hubble's law and to revise the basics of modern cosmology. Compared to the Big bang model, advantage of Black hole cosmology lies in confirming its validity through atomic and nuclear experimental results.

\section{Black Holes and Black Hole Cosmology}

In the standard cosmology, 'Hubble volume' or 'Hubble sphere' is a spherical region of the Universe surrounding an observer beyond which objects recede from that observer at a rate greater than the speed of light due to the expansion of the Universe. The commoving radius of a Hubble sphere (known as the Hubble radius or the Hubble length) is $\left(c / H_{0}\right)$, where $(c)$ is the speed of light and $\left(H_{0}\right)$ is the Hubble constant. More generally, the term 'Hubble volume' can be applied to any region of space with a volume of the order of $(4 \pi / 3)\left(c / H_{0}\right)^{3}$

. 'Hubble volume' can be considered as a key tool in cosmology and unification. Please note that at any given cosmic time, the product of 'cosmic critical density' and 'Hubble volume' gives a characteristic cosmic mass and it can be called as the 'Hubble mass'. Interesting thing is that, Schwarzschild radius of the 'Hubble mass' again matches with the 'Hubble length'. Most of the cosmologists believe that this is merely a coincidence. Here the authors emphasize the fact that this coincidence is having deep connection with the cosmic geometry and the cosmological \& microscopic physical phenomena. At any given cosmic time, 'Hubble length' can be considered as the gravitational or electromagnetic interaction range. If one is willing to think in this direction, by increasing the number of applications of Hubble mass and Hubble volume in other areas of fundamental physics like quantum physics, nuclear physics, atomic physics and particle physics slowly and gradually - in a progressive way, concepts of 'Black hole Cosmology' can be strengthened and can also be confirmed [17-21]. If one is able to show the applications of 'Hubble mass' in different areas of fundamental physics, certainly it can be given more significance and top priority compared to the mysterious 'dark energy'.

With reference to the well believed big bang, in the universe there is no centre, there is no preferred direction and there is no rotation. With reference to galactic spinning black holes, it is well confirmed 
that, there is a center, there is rotation and there is a preferred direction. Considering a $4 \mathrm{D} / 3 \mathrm{D}$ or $3 \mathrm{D}$ star like black hole (that is assumed to be responsible for the cosmic evolution) with no centre, with no preferred direction and with no rotation is not correct. Hence the possible 'new solution' seems to be - to give up the old unanswerable concepts of big bang and to become accustomed with the newly accepted concepts of $4 \mathrm{D} / 3 \mathrm{D}$ or $3 \mathrm{D}$ cosmic primordial black hole with center and rotation and see the consequences!

\section{The Proposed Picture of Black Hole Cosmology}

Instead of considering 'star - black hole explosions' and 'higher dimensions', the authors of this paper focused their attention only on the old and famous Mach's principle, 'Hubble volume' and 'primordial evolving black holes'. Some cosmologists use the term 'Hubble volume' to refer to the volume of the observable universe. There is no perfect theory that defines the lower and upper limits of a massive black hole. Most of the theoretical models assume a lower mass limit close to the 'Planck mass'. Astronomers believe that black holes that are as large as a billion solar masses can be found at the centre of most of the galaxies. Here the fundamental questions to be answered are: If the galactic central black hole mass is 10 billion solar masses and density is less than $1 \mathrm{~kg} / \mathrm{m}^{3}$ - with such a small density and large mass, without collapsing how it is able to hold a gigantic galaxy? What force makes the black hole stable? Recent observations confirm that, instead of collapsing, galactic central black holes are growing faster and spinning with light speed. Even though mass is too high and density is too low, light speed rotation certainly helps in maintaining black hole's stability from collapsing with maximum possible outward radial force of the magnitude close to $\left(c^{4} / G\right)$.If 'black hole geometry' is intrinsic compared to the black hole 'mass' and 'density' parameters, if universe constitutes so many galaxies and if each galaxy constitutes a central growing and fast spinning black hole then considering universe as an 'evolving and light speed rotating primordial black hole' may not be far away from reality.

Based on these points the authors propose the following picture of Black hole cosmology. Forever rotating at light speed, high temperature and high angular velocity small sized primordial cosmic black hole of mass $M_{C} \cong \sqrt{e^{2} / 4 \pi \varepsilon_{0} G}$ gradually transforms into a low temperature and low angular velocity large sized massive primordial cosmic black hole. At any given cosmic time, for the primordial growing black hole universe, its 'Schwarzschild radius' can be considered as its characteristic possible minimum radius and 'constant light speed rotation' will give the maximum possible stability from collapsing. Here $M_{C} \cong \sqrt{e^{2} / 4 \pi \varepsilon_{0} G}$ can be called as the mass of the primordial baby black hole universe. Here 3 important points can be stated as follows.

In theoretical physics, particularly in discussions of gravitation theories, Mach's principle is the name given by Einstein to an interesting hypothesis often credited to the physicist and philosopher Ernst Mach. The idea is that the local motion of a rotating reference frame is determined by the large scale distribution of matter. With reference to the Mach's principle and the Hubble volume, at any cosmic time, if 'Hubble mass' is the product of cosmic 'critical density' and the 'Hubble volume', then it can be suggested that, i) Each and every point in the free space is influenced by the Hubble mass, ii) Hubble volume and Hubble mass play a vital role in understanding the properties of electromagnetic and nuclear interactions and iii) Hubble volume and Hubble mass play a key role in understanding the geometry of the universe. As a first attempt, in this paper authors proposed a semi empirical relation that connects the CMBR energy density, Hubble's constant and $\sqrt{e^{2} / 4 \pi \varepsilon_{0} G}$.

Starting from an electron to any gigantic galaxy, rotation is a common phenomenon in atomic experiments and astronomical observations. From Newton's laws of motion and based on the Mach's principle, sitting inside a closed universe, one cannot comment whether the universe is rotating or not. We have to search for alternative means for confirming the cosmic rotation. Recent findings from the University of Michigan [22] suggest that the shape of the Big Bang might be more complicated than previously thought, and that the early universe spun on an axis. A left-handed and right-handed imprint on the sky as reportedly revealed by galaxy rotation would imply the universe was rotating from the very beginning and retained an overwhelmingly strong angular momentum. An anonymous referee who reviewed the paper for Physics Letters said, "In the paper the author claims that there is a preferred handedness of spiral galaxies indicating a preferred direction in the universe. Such a claim, if proven true, would have a profound impact on cosmology and would very likely result in a "Nobel prize". The consequences of a spinning universe [22-36] seem to be profound and natural. Not only that, with 'constant rotation speed' 'cosmic collapse' can be prevented and can be considered as an alternative to the famous 'repulsive gravity' concept. If so, at any time to have maximum possible stability from collapsing 'constant light speed rotation' can be considered as a constructive and workable concept.

Recent observations confirm black hole's light speed rotation. In 2013 February, using NASA's newly launched NuStar telescope and the European Space Agency's workhorse XMM-Newton, an international team observed high-energy X-rays released by a super massive black hole in the middle of a nearby galaxy. They calculated its spin at close to the speed of light: 670 million mph [37]. Please note that, for any black hole even though its mass is too high and density is too low, light speed rotation certainly helps in maintaining its stability from collapsing with maximum possible outward radial force [3] of magnitude $\left(c^{4} / G\right)$. At the beginning of comic evolution if rotation speed was zero and there was no big bang - definitely it will cast a doubt on the stability, existence and angular velocity of the assumed initial primordial cosmic baby black hole. Hence at the beginning also, to guess or define the angular velocity and to have maximum possible stability it is better to assume light speed rotation for the cosmic baby black hole. At present if rate of cosmic expansion is very slow, then rate of decrease in angular velocity will be very small and practically can be considered as zero. Along with (practically) constant angular velocity, at present if constant light speed rotation is assumed to be maintained then cosmic stability will be maximum and rate of change in cosmic size will be practically zero and hence this idea helps us to believe in present Hubble length along with the observed ordered galactic structures and uniform thermal energy density.

\section{The Cosmic 'Critical Density' and its Dimensional Analysis and the Cosmic Rotation}

With a simple derivation it is possible to show that, Hubble's constant $H_{t}$ represents cosmological angular velocity. Authors presented this derivation in their published papers. Basic idea of this derivation is to express the angular velocity of any rotating celestial body in terms of its mass, radius, mass density and surface escape velocity.

Assume that, a planet of mass $\mathrm{M}$ and radius $\mathrm{R}$ rotates with angular velocity $\omega_{e}$ and linear velocity $v_{e}$ in such a way that, free or loosely bound particle of mass $m$ lying on its equator gains a kinetic energy equal to potential energy as,

$$
\frac{1}{2} m v_{e}^{2}=\frac{G M m}{R}
$$




$$
R \omega_{e}=v_{e}=\sqrt{\frac{2 G M}{R}} \text { and } \omega_{e}=\frac{v_{e}}{R}=\sqrt{\frac{2 G M}{R^{3}}}
$$

i.e Linear velocity of planet's rotation is equal to free particle's escape velocity. Without any external power or energy, test particle gains escape velocity by virtue of planet's rotation. Note that if Earth completes one rotation in one hour then free particles lying on the equator will get escape velocity. Now writing $M=\frac{4 \pi}{3} R^{3} \rho_{e}$,

$$
\begin{aligned}
& \omega_{e}=\frac{v_{e}}{R}=\sqrt{\frac{8 \pi G \rho_{e}}{3}} \text { Or } \omega_{e}^{2}=\frac{8 \pi G \rho_{e}}{3} \\
& \text { Density, } \rho_{\mathrm{e}}=\frac{3 \omega_{\mathrm{e}}^{2}}{8 \pi \mathrm{G}}
\end{aligned}
$$

In real time, this obtained density may or may not be equal to the actual density. But the ratio $\frac{8 \pi G \rho_{\text {ral }}}{3 \omega_{\text {wel }}}$ may have some physical significance. The most important point to be noted here, is that, as far as dimensions and units are considered, from equation (4), it is very clear that, proportionality constant being $\frac{3}{8 \pi G}$, density $\propto(\text { angular velocity })^{2}$

Equation (4) is similar to "flat model concept" of cosmic "critical density"

$$
\rho_{c}=\frac{3 H_{t}^{2}}{8 \pi G}
$$

Comparing equations (4) and (6) dimensionally and conceptually, i.e.

$$
\begin{aligned}
& \rho_{e}=\frac{3 \omega_{e}^{2}}{8 \pi G} \text { with } \rho_{\mathrm{c}}=\frac{3 H_{\mathrm{t}}^{2}}{8 \pi \mathrm{G}} \\
& H_{t}^{2} \rightarrow \omega_{\mathrm{e}}^{2} \text { and } H_{t} \rightarrow \omega_{e}
\end{aligned}
$$

It is very clear that, dimensions of 'Hubble's constant' must be 'radian/second'. In any physical system under study, for any one 'simple physical parameter' there will not be two different units and there will not be two different physical meanings. This is a simple clue and brings 'cosmic rotation' into picture. This is possible in a closed universe only. Cosmic models that depend on this "critical density" may consider 'angular velocity of the universe' in the place of 'Hubble's constant'. In the sense, with a great confidence 'cosmic rotation' can be included in the existing models of cosmology. Then the term 'critical density' appears to be the 'volume density' of the closed and expanding universe.

\section{To Re Interpret the Hubble's Law}

It may be noted that, increased redshifts and increased distances forced Edwin Hubble to propose the Hubble's law. Since galaxy is not a point particle and if light is coming from the atoms of the gigantic galaxy, then cosmic redshift can be interpreted as an index of the galactic atomic 'light emission mechanism'. In no way it seems to be connected with 'galaxy receding. If it is possible to show that, (from the observer) observed older galaxy's distance increases with its 'age', then the concepts 'galaxy receding' and 'accelerating universe' can be put for a revision at fundamental level. Whatever may be the expression, definitions of cosmic red shift seem to be ad-hoc and not absolute. With reference to our laboratory or our galaxy, the basic or original definition of present/current redshift $\left(z_{0}\right)$ can be expressed as follows.

$$
z_{0} \cong \frac{E_{0}-E_{G}}{E_{0}} \cong \frac{\lambda_{G}-\lambda_{0}}{\lambda_{G}} \cong\left(z_{x}\right)_{0} \leq 1 \text {. (say) }
$$

But not

$$
z_{0} \cong \frac{E_{0}-E_{G}}{E_{G}} \cong \frac{\lambda_{G}-\lambda_{0}}{\lambda_{0}} \cong\left(z_{y}\right)_{0} \text { (say) }
$$

With reference to the current definition of $z \cong\left(z_{y}\right)_{0}$, proposed $z \cong\left(z_{x}\right)_{0}$ can be expressed as follows.

$$
\left(z_{x}\right)_{0} \cong \frac{\left(z_{y}\right)_{0}}{1+\left(z_{y}\right)_{0}}
$$

Here $E_{0} \cong \frac{h c}{\lambda_{0}}$ is the energy of photon at our galaxy/laboratory and $E_{G} \cong \frac{h c}{\lambda_{G}}$ is the energy of received photon when it was emitted in the galaxy. Similarly $\lambda_{G}$ is the wave length of light received from distant galaxy when it was emitted and $\lambda_{0}$ is the wave length of light in laboratory. Even though both relations are ad-hoc and not absolute definitions, compared to relation (10), relation (9) seems to be somewhat reliable. Very interesting thing is that, when redshift is very small (up to $z \approx 0.01$ ), both relations almost all will give the same result. Important point to be noticed is that, by Hubble's time the maximum redshift noticed was 0.003 and was less than 0.01 . One should not ignore this fact. Now the fundamental question to be answered is: which relation is correct: either relation (9) or relation (10)? Note that, present red shift $\left(z_{0}\right)$ will be directly proportional to age difference between our galaxy and observed galaxy or time taken by light to reach our galaxy from the observed galaxy $(\Delta t)$. Thus $z_{0} \propto \Delta t$ and

$$
z_{0} \cong H_{0} \Delta t \text {. }
$$

Here $H_{0}$ is the proportionality constant. In this way $H_{0}$ can be incorporated directly. Time taken by light to reach our galaxy or the age difference of our galaxy and observed galaxy can be expressed as,

$$
\begin{aligned}
& \Delta t \cong \frac{z_{0}}{H_{0}} . \\
& c \Delta t \cong z_{0} \cdot \frac{c}{H_{0}} .
\end{aligned}
$$

To confirm this, absolute methods (that are free from redshift) for estimating galaxy age can be considered. Then the basic and original definition of 'galaxy receding' and 'accelerating universe' concepts can be eliminated and a 'decelerating or expanded universe' concept can be continued without any difficulty. Hence with redshift concept - one may not be able to understand the actual rate of cosmic expansion and actual cosmic geometry [38,39].

\section{Possible Assumptions}

The possible assumptions in unified cosmic physics can be expressed in the following way.

A) With reference to the elementary charge and with mass similar to the Planck mass, a new mass unit can be constructed in the following way. It can be called as the Coulomb mass.

$$
\begin{aligned}
\left(M_{C}\right)^{ \pm} & \cong \sqrt{\frac{e^{2}}{4 \pi \varepsilon_{0} G}} \cong 1.859210775 \times 10^{-9} \mathrm{Kg} \\
& \cong 1.042941 \times 10^{18} \mathrm{GeV} / \mathrm{c}^{2}
\end{aligned}
$$

It is well known that $e, c$ and $G$ play a vital role in fundamental physics. With these 3 constants space-time curvature concepts at a charged particle surface can be studied. Note that the basic concept of unification is to understand the origin of 'mass' of any particle. Mass is the basic property in 'gravitation' and charge is the basic property in 'atomicity'. So far no model established a cohesive relation in between 'electric charge' and 'mass' of any 'elementary particle' or 'cosmic dust'. 
From physics point of view, the fundamental questions to be answered are: 1) Without charge, is there any independent existence to "mass"? 2) Without mass, is there any independent existence to "charge"? From cosmology point of view the fundamental questions to be answered are: 1)What is 'cosmic dust'? 2) Without charge, is there any independent existence to "cosmic dust"? From astrophysics point of view the fundamental questions to be answered are: 1) Without charge, is there any independent existence to 'mass' of any star? 2) Is black hole - a neutral body or electrically a neutralized body? To understand these questions the authors made an attempt to construct the above unified mass unit. With this mass unit in unification program with a suitable proportionality it may be possible to represent the characteristic mass of elementary charge. It can be considered as the seed of galactic matter or galactic central black hole. It can also be considered as the seed of any cosmic structure. If 2 such oppositely charged particles annihilates, a large amount of energy can be released.

At any time Hubble length $\left(c / H_{t}\right)$ can be considered as the gravitational or electromagnetic interaction range.

At any time, $H_{t}$ being the angular velocity, universe can be considered as a growing and light speed rotating primordial black hole. Thus at any given cosmic time,

$$
R_{t} \cong \frac{2 G M_{t}}{c^{2}} \cong \frac{c}{H_{t}} \text { and } M_{t} \cong \frac{c^{3}}{2 G H_{t}}
$$

when $M_{t} \rightarrow M_{C}, R_{C} \cong \frac{2 G M_{C}}{c^{2}}$ and $H_{C} \cong \frac{c}{R_{C}} \cong \frac{c^{3}}{2 G M_{C}}$ can be considered as the characteristic initial physical measurements of the universe. Here the subscript $C$ refers to the initial conditions of the universe and can be called as the Coulomb scale. Similarly $R_{0} \cong \frac{2 G M_{0}}{c^{2}} \cong \frac{c}{H_{0}}$ and $\mathrm{M}_{0} \cong \frac{c^{3}}{2 G H_{0}}$ can be considered as the characteristic current physical measurements of the universe.

B) Cosmic time is real and absolute.

\section{Connecting Cosmic Thermal and Physical Parameters}

It may be noted that connecting CMBR energy density with Hubbles' constant is really a very big task and mostly preferred in cosmology. At any given cosmic time, thermal energy density can be expressed with the following semi empirical relation.

$$
\begin{gathered}
a T_{t}^{4} \cong\left[1+\ln \left(\frac{M_{t}}{M_{C}}\right)\right]^{-2}\left(\frac{3 H_{t}^{2} c^{2}}{8 \pi G}\right) \\
\cong\left[1+\ln \left(\frac{H_{C}}{H_{t}}\right)\right]^{-2}\left(\frac{3 H_{t}^{2} c^{2}}{8 \pi G}\right)
\end{gathered}
$$

With a suitable derivation if above expression is obtained, then certainly the subject of black hole cosmology is put into main stream physics. At any time

$$
\frac{3 H_{t}^{2} c^{2}}{8 \pi G a T_{t}^{4}} \cong\left[1+\ln \left(\frac{M_{t}}{M_{C}}\right)\right]^{2} \cong\left[1+\ln \left(\frac{H_{C}}{H_{t}}\right)\right]^{2}
$$

Thus at present, if $H_{0}$ is close to $71 \mathrm{~km} / \mathrm{sec} / \mathrm{Mpc}$, obtained CMBR temperature is $2.723{ }^{0} \mathrm{~K}$. For the time being this can be considered as a remarkable discovery and an accurate fit.

$$
\begin{gathered}
a T_{0}^{4} \cong\left[1+\ln \left(\frac{H_{C}}{H_{t}}\right)\right]^{-2}\left(\frac{3 H_{0}^{2} c^{2}}{8 \pi G}\right) \\
\cong\left[1+\ln \left(\frac{M_{0}}{M_{C}}\right)\right]^{-2}\left(\frac{3 H_{0}^{2} c^{2}}{8 \pi G}\right)
\end{gathered}
$$

Mostly at the ending stage of expansion, rate of change in $H_{t}$ will be practically zero and can be considered as practically constant. Thus at its ending stage of expansion, for the whole cosmic black hole as $H_{t}$ practically remains constant, its corresponding thermal energy density will be 'the same' throughout its volume. This 'sameness' may be the reason for the observed 'isotropic' nature of the current $\mathrm{CMB}$ radiation [40-43]. With this coincidence it can be suggested that, at the beginning of cosmic evolution,

$$
a T_{C}^{4} \cong\left(\frac{3 H_{C}^{2} c^{2}}{8 \pi G}\right)
$$

Based on the rate of decrease in $\frac{d}{d t}\left(T_{t}\right)$ and $\frac{d}{d t}\left(H_{t}\right)$ at any time, the absolute cosmic rate of expansion be confirmed. Matter-energy density can be considered as the geometric mean density of volume energy density and the thermal energy density and it can be expressed with the following semi empirical relation.

$$
\begin{aligned}
& \left(\rho_{m}\right)_{t} c^{2} \cong \sqrt{\left(\frac{3 H_{t}^{2} c^{2}}{8 \pi G}\right)\left(a T_{t}^{4}\right)} \cong\left[1+\ln \left(\frac{H_{C}}{H_{t}}\right)\right]^{-1}\left(\frac{3 H_{t}^{2} c^{2}}{8 \pi G}\right) \\
& \cong\left[1+\ln \left(\frac{M_{t}}{M_{C}}\right)\right]^{-1}\left(\frac{3 H_{0}^{2} c^{2}}{8 \pi G}\right)
\end{aligned}
$$

Here one important observation to be noted is that, at any time

$$
\frac{3 H_{t}^{2}}{8 \pi G\left(\rho_{m}\right)_{t}} \cong\left[1+\ln \left(\frac{M_{t}}{M_{C}}\right)\right] \cong\left[1+\ln \left(\frac{H_{C}}{H_{t}}\right)\right]
$$

Thus at present,

$$
\begin{aligned}
& \left(\rho_{m}\right)_{0} \cong \frac{1}{c^{2}} \sqrt{\left(\frac{3 H_{0}^{2} c^{2}}{8 \pi G}\right)\left(a T_{0}^{4}\right) \cong\left[1+\ln \left(\frac{H_{C}}{H_{0}}\right)\right]^{-1}\left(\frac{3 H_{0}^{2}}{8 \pi G}\right)} \\
& \cong\left[1+\ln \left(\frac{M_{0}}{M_{C}}\right)\right]^{-1}\left(\frac{3 H_{0}^{2}}{8 \pi G}\right) \cong 6.6 \times 10^{-32} \mathrm{gram} / \mathrm{cm}^{3}
\end{aligned}
$$

Based on the average mass-to-light ratio for any galaxy present matter density can be expressed with the following relation [44] $\left(\rho_{m}\right)_{0} \cong 1.5 \times 10^{-32} \eta h_{0}$ gram $/ \mathrm{cm}^{3}$.

Here $\eta \cong\left\langle\frac{M}{L}\right\rangle_{\text {galaxx }} /\left\langle\frac{M}{L}\right\rangle_{\text {sum }}, h_{0} \cong H_{0} / 100 \mathrm{Km} / \mathrm{sec} / \mathrm{Mpc} \cong 0.71$ Note that elliptical galaxies probably comprise about $60 \%$ of the galaxies in the universe and spiral galaxies thought to make up about $20 \%$ percent of the galaxies in the universe. Almost $80 \%$ of the galaxies are in the form of elliptical and spiral galaxies. For spiral galaxies, $\eta h_{0}^{-1} \cong 9 \pm 1$ and for elliptical galaxies, $\eta h_{0}^{-1} \cong 10 \pm 2$ For our galaxy inner part, $\eta h_{0}^{-1} \cong 6 \pm$ 2. Thus the average $\eta h_{0}^{-1}$ is very close to 8 to 9 and its corresponding matter density is close to $(6.0$ to 6.7$) \times 10^{-32} \mathrm{gram} / \mathrm{cm}^{3}$ and can be compared with the above proposed magnitude of $6.6 \times 10^{-32} \mathrm{gram} / \mathrm{cm}^{3}$.

Somehow and by any reason, magnitude of the current mass of the black hole universe being same, at present if volume density approaches to the current matter density, then cosmic radius increases by a factor 5.2.Similarly if volume density approaches to the current thermal energy density, then cosmic radius increases by a factor 27.3. These two numbers can be compared with the presently believed first two of the three cosmological numbers $4.9 \%, 26.8 \%$ and $68.3 \%$. Thinking in this way from cosmology point of view logically 'dark matter' and 'dark energy' concepts can be put for a revision at elementary level. 


\section{Direct Fitting of the Current CMBR Wave Length}

Note that the spectrum from Planck's law of black body radiation takes a different shape in the frequency domain from that of the wavelength domain, the frequency location of the peak emission does not correspond to the peak wavelength using the simple relationship between frequency, wavelength, and the speed of light. In other words, the peak wavelength and the peak frequency do not correspond. The frequency form of Wien's displacement law is derived using similar methods, but starting with Planck's law in terms of frequency instead of wavelength. The effective result is to substitute 3 for 5 in the equation for the peak wavelength. Thus it is possible to say that [45],

$$
\sqrt{\frac{c}{\lambda_{m} f_{m}}} \cong \sqrt{1.75978} \cong 1.326567 \cong \frac{4}{3}
$$

where $\lambda_{m}$ and $f_{m}$ are the peak wavelength in wavelength domain and peak frequency in frequency domain respectively.

Let $\lambda_{f}$ is the wavelength corresponding to $\frac{d E_{v}}{d v}$ and $E_{v}$ is the total energy at all frequencies up to and including $v$, at any given cosmic time. $\lambda_{m}$ is the wavelength corresponding to $\frac{d E_{\lambda}}{d \lambda}$ and $E_{\lambda}$ is the total energy at all wavelengths up to and including $\lambda^{d \lambda}$.

Considering the observed CMBR wavelengths, it is possible to express both the wavelengths in the following way.

$$
\begin{aligned}
& {\left[\left(\lambda_{m}\right)_{t} \text { and }\left(\lambda_{f}\right)_{t}\right] \propto \sqrt{1+\ln \left(\frac{M_{t}}{M_{C}}\right)}} \\
& {\left[\left(\lambda_{m}\right)_{t} \text { and }\left(\lambda_{f}\right)_{t}\right] \propto \sqrt{\left(\frac{4 \pi G M_{t}}{c^{2}}\right) \cdot\left(\frac{4 \pi G M_{C}}{c^{2}}\right)}}
\end{aligned}
$$

Guessing in this way it is noticed that,

$$
\begin{aligned}
& \left(\lambda_{f}\right)_{t} \cong\left(\frac{4}{3}\right) \cdot \sqrt{1+\ln \left(\frac{M_{t}}{M_{C}}\right)} \cdot \frac{4 \pi G \sqrt{M_{t} M_{C}}}{c^{2}} \\
& \left(\lambda_{m}\right)_{t} \cong\left(\frac{3}{4}\right) \cdot \sqrt{1+\ln \left(\frac{M_{t}}{M_{C}}\right)} \cdot \frac{4 \pi G \sqrt{M_{t} M_{C}}}{c^{2}}
\end{aligned}
$$

Thus it is possible to express both the wavelength relations in the following way.

$$
\begin{aligned}
& \left(\lambda_{f}, \lambda_{m}\right)_{t} \cong\left(\frac{4}{3}\right)^{ \pm 1} \cdot \sqrt{1+\ln \left(\frac{M_{t}}{M_{C}}\right)} \cdot \frac{4 \pi G \sqrt{M_{t} M_{C}}}{c^{2}} \\
& \cong\left(\frac{4}{3}\right)^{ \pm 1} \cdot \sqrt{1+\ln \left(\frac{H_{C}}{H_{t}}\right)} \cdot \frac{2 \pi c}{\sqrt{H_{C} H_{t}}}
\end{aligned}
$$
follows.

Alternatively geometric mean of $\left(\lambda_{f}, \lambda_{m}\right)_{t}$ can be expressed as

$$
\begin{gathered}
\sqrt{\left(\lambda_{m}\right)_{t}\left(\lambda_{f}\right)_{t}} \cong \sqrt{1+\ln \left(\frac{M_{t}}{M_{C}}\right) \cdot \frac{4 \pi G \sqrt{M_{t} M_{C}}}{c^{2}}} \\
\cong \sqrt{1+\ln \left(\frac{H_{C}}{H_{t}}\right)} \cdot \frac{2 \pi c}{\sqrt{H_{C} H_{t}}}
\end{gathered}
$$

At present, if $H_{0}$ is close to $71 \mathrm{~km} / \mathrm{sec} / \mathrm{Mpc}$,

$$
\begin{aligned}
& \left(\lambda_{f}, \lambda_{m}\right)_{0} \cong\left(\frac{4}{3}\right)^{ \pm 1} \cdot \sqrt{1+\ln \left(\frac{M_{0}}{M_{C}}\right)} \cdot \frac{4 \pi G \sqrt{M_{0} M_{C}}}{c^{2}} \\
& \cong\left(\frac{4}{3}\right)^{ \pm 1} \cdot \sqrt{1+\ln \left(\frac{H_{C}}{H_{0}}\right)} \cdot \frac{2 \pi c}{\sqrt{H_{C} H_{t}}} \\
& \cong(1.90 \mathrm{~mm}, 1.069 \mathrm{~mm})
\end{aligned}
$$

With reference to $\left(\lambda_{m}\right)_{t}$ and Wien's displacement constant, from relation (28) $k_{B} T_{t}$ can be expressed as follows.

$$
\begin{aligned}
& T_{t} \cong \frac{2.898 \times 10^{-3}}{\left(\lambda_{m}\right)_{t}} \cong\left(\frac{h c}{4.965114 k_{B}}\right)\left(\frac{1}{\left(\lambda_{m}\right)_{t}}\right) \text { and } \\
& k_{B} T_{t} \cong\left(\frac{4}{3 x}\right) \sqrt{\left(1+\ln \left(\frac{M_{t}}{M_{C}}\right)\right)^{-1}\left(\frac{M_{t}}{M_{C}}\right)} \cdot\left(\frac{h c^{3}}{4 \pi G M_{t}}\right)
\end{aligned}
$$

where $x \cong 4.965114$

$$
k_{B} T_{t} \propto\left(\frac{h c^{3}}{4 \pi G M_{t}}\right) \cong \frac{h H_{t}}{2 \pi}
$$

This relation may not be identical but similar to the famous Hawking's black hole temperature formula [46].

$$
k_{B} T_{t} \propto \sqrt{\left(1+\ln \left(\frac{M_{t}}{M_{C}}\right)\right)^{-1}\left(\frac{M_{t}}{M_{C}}\right)}
$$

In this way in a very simple approach observed CMBR and the proposed Black hole universe concepts can be put into single frame of reference. Here the very interesting and strange observation is that, at present

$$
\left(1+\ln \left(\frac{M_{0}}{M_{C}}\right)\right)^{-1}\left(\frac{M_{0}}{M_{C}}\right) \cong \exp \left(\frac{1}{\alpha}\right)
$$

where $\left(\frac{1}{\alpha}\right)$ is the inverse of the fine structure ratio. For any mathematician this seems be a fun .For a cosmologist it may be an accidental coincidence. For any physicist it is an astounding and exciting coincidence. Even though it depends upon one's own choice of scientific interest, from unification point of view, assuming it to be a cosmological variable it is possible to express $\left(\frac{1}{\alpha}\right)$ in the following way.

$$
\left(\frac{1}{\alpha}\right)_{0} \cong \ln \left[\left(1+\ln \left(\frac{M_{0}}{M_{C}}\right)\right)^{-1}\left(\frac{M_{0}}{M_{C}}\right)\right] \cong 137.047
$$

Here $\left(\frac{1}{\alpha}\right)_{0}$ may be considered as the current magnitude of 'inverse of the fine structure ratio. Based on this heuristic observation and for the assumed initial conditions of the universe, if $M_{t} \rightarrow M_{C},\left(\frac{1}{\alpha}\right)_{C} \rightarrow 0$.

Now the fundamental questions to be answered are -

1) Is Fine structure ratio - a cosmological variable?

2) Is the reduced Planck's constant - a cosmological variable?

3) Is the Planck's constant - a cosmological constant?

4) How to understand and how to consider the constancy of the Planck's constant along with the variable reduced Planck's constant?

5) Is reduced Planck's constant - an output of the atomic system?

Based on the relation (35), if one is willing to consider the cosmological variable nature of $\left(\frac{1}{\alpha}\right)$, relation (32) can be expressed as follows. 


$$
T_{t} \cong \sqrt{\left(e^{\frac{1}{\alpha}}\right)_{t}} \cdot\left(\frac{b c^{2}}{3 \pi G M_{t}}\right)
$$

At the beginning of cosmic evolution,

$$
T_{C} \cong\left(\frac{b c^{2}}{3 \pi G M_{C}}\right)
$$

From ground based laboratory experiments, it is possible to measure the rate of change $\frac{d}{d t}\left(\frac{1}{\alpha_{t}}\right)$. Hence the absolute cosmic rate of expansion can be measured and thus the presently believed 'accelerating model' of cosmology can be verified. In this direction it is noticed that,

$$
\frac{8 \pi^{5}}{15}\left(\frac{b k_{B}}{h c}\right)^{3} \cong \frac{4}{3}
$$

From relations $(20,24,38,39)$ the Boltzmann's constant and Wien's displacement constant can be interrelated with the elementary charge in the following way.

$$
b \cong \frac{729 \pi^{3}}{128}\left(\frac{e^{2}}{4 \pi \varepsilon_{0} k_{B}}\right) \cong 2.95084 \times 10^{-3}{ }^{0} \mathrm{~K} . \mathrm{m}
$$

Here accuracy is close to $98.18 \%$. Thus

$$
\begin{aligned}
h \cong\left[\left(\frac{2 \pi^{5}}{5}\right)^{\frac{1}{3}}\left(\frac{729 \pi^{3}}{128}\right)\right]\left(\frac{e^{2}}{4 \pi \varepsilon_{0} c}\right) \\
\cong 6.7475333 \times 10^{-34} \mathrm{~J} . \mathrm{sec}
\end{aligned}
$$

\section{The Cosmic Redshift and its New Interpretation}

Observed cosmic red shift can be reinterpreted as a cosmological galactic atomic light emission mechanism. If one is willing to consider this proposal, in hydrogen atom emitted photon energy can be understood as follows.

1. During cosmic evolution, as cosmic time increases, hydrogen atom emits photons with increased quanta of energy. Thus past light quanta emitted from old galaxy will have less energy and show a red shift with reference to our galaxy.

2. During journey light quanta will not lose energy and there will be no change in light wavelength.

3. Galactic photon energy when it was emitted can be estimated as follows.

$$
E_{t} \cong\left(\frac{\lambda_{0}}{\lambda_{G}}\right)\left(\frac{h c}{\lambda_{0}}\right) \cong \frac{h c}{\lambda_{G}}
$$

Here, $\lambda_{0}$ is the wavelength of photon in the laboratory.

$E_{t}$ is the energy of received photon when it was emitted in the distant galaxy.

$\lambda_{G}$ the wavelength of received photon when it was emitted in the distant galaxy.

In the following section an attempt is made to understand the cosmological atomic light emission mechanism in hydrogen atom.

\section{Cosmological Discrete Bohr Radii, Discrete Force, Discrete Potential and Discrete Nature of Angular Momentum in Hydrogen Atom}

Note that, in any bound system, 'operating force' only plays a major role in maintaining the 'existence of the bound system' and 'angular momentum' is one of the results. If one is able to make the operating force as discrete, then automatically one can observe a discrete structure like discrete radii, discrete angular momentum and discrete energy levels. The assumed cosmological characteristic discrete operating force can be expressed as follows.

$$
\begin{aligned}
& \left(F_{X}\right)_{n} \cong\left(\frac{c^{4}}{n G}\right) \cong \frac{1}{n}\left(\frac{c^{4}}{G}\right) \text { Or } \\
& \left(F_{Y}\right)_{n} \cong\left(\frac{c^{4}}{n^{2} G}\right) \cong \frac{1}{n^{2}}\left(\frac{c^{4}}{G}\right)
\end{aligned}
$$

where $n=1,2,3 \ldots$ Note that $\left(c^{4} / G\right)$ can be considered as the limiting magnitude of any kind of force. Similarly $\left(c^{5} / G\right)$ can be considered as the limiting magnitude of any kind of power $[1,2,3]$. Based on this proposal, the characteristic angular momentum can be shown to be proportional to $n$ or $\sqrt{n}$. Vector sum of $n$ and $\sqrt{n}$ can be expressed as follows

$$
\sqrt{(n)^{2}+(\sqrt{n})^{2}} \cong \sqrt{n^{2}+n}=\sqrt{n(n+1)} .
$$

In a cosmological approach with various trial-error methods, at present in hydrogen atom, Bohr radius can be fitted as follows.

$$
\left(a_{B}\right)_{0} \cong\left(\frac{4 \pi \varepsilon_{0} G m_{p}^{2}}{e^{2}}\right)\left(\frac{G M_{0}}{c^{2}}\right) \cong\left(\frac{4 \pi \varepsilon_{0} M_{0} c^{2}}{e^{2}}\right)\left(\frac{G m_{p}}{c^{2}}\right)^{2}
$$

Note that, this relation is free from the famous reduced Planck's constant, electron rest mass and other arbitrary numbers or coefficients. With reference to the proposed discrete force and from above observation/fitting, current Bohr radii can be expressed as follows.

$$
n^{2}\left(a_{B}\right)_{0} \cong\left(\frac{4 \pi \varepsilon_{0} M_{0} c^{2}}{e^{2}}\right)\left(G m_{p}^{2}\right)\left(\frac{n^{2} G}{c^{4}}\right)
$$

In the past,

$$
\begin{aligned}
& n^{2}\left(a_{B}\right)_{t} \cong\left(\frac{M_{0}}{M_{t}}\right)\left(\frac{4 \pi \varepsilon_{0} M_{0} c^{2}}{e^{2}}\right)\left(G m_{p}^{2}\right)\left(\frac{n^{2} G}{c^{4}}\right) \\
& \left(a_{B}\right)_{t} \propto\left(G m_{p}^{2}\right) \\
& \left(a_{B}\right)_{t} \propto\left(\frac{e^{2}}{4 \pi \varepsilon_{0} M_{0} c^{2}}\right)^{-1} \\
& \left(a_{B}\right)_{t} \propto\left(\frac{c^{4}}{n^{2} G}\right)^{-1} \\
& \left(a_{B}\right)_{t} \propto\left(\frac{M_{0}}{M_{t}}\right)^{2}
\end{aligned}
$$

With reference to $n^{2}$ form, the current unified cosmological potential in hydrogen atom can be expressed as follows.

$$
\left(E_{\mathrm{pot}}\right)_{0} \cong-\left(\frac{e^{2}}{4 \pi \varepsilon_{0} G m_{p}^{2}}\right)\left(\frac{e^{2}}{4 \pi \varepsilon_{0} M_{0} c^{2}}\right)\left(\frac{c^{4}}{n^{2} G}\right)
$$

If revolving electron's kinetic energy is equal to half the magnitude of potential energy, then 


$$
\left(E_{\text {kin }}\right)_{0} \cong \frac{1}{2}\left(\frac{e^{2}}{4 \pi \varepsilon_{0} G m_{p}^{2}}\right)\left(\frac{e^{2}}{4 \pi \varepsilon_{0} M_{0} c^{2}}\right)\left(\frac{c^{4}}{n^{2} G}\right)
$$

At present in hydrogen atom, emitted photon energy can be expressed as follows.

$$
\begin{aligned}
\left(E_{\text {photon }}\right)_{0} & \cong \frac{1}{2}\left(\frac{e^{2}}{4 \pi \varepsilon_{0} G m_{p}^{2}}\right)\left(\frac{e^{2}}{4 \pi \varepsilon_{0} M_{0} c^{2}}\right)\left[\left(\frac{c^{4}}{G}\right)\left(\frac{1}{n_{1}^{2}}-\frac{1}{n_{2}^{2}}\right)\right] \\
& \cong \frac{h c}{\lambda_{0}}
\end{aligned}
$$

where $n_{1}=n_{2}=1,2,3 .$, and $\mathrm{n}_{2}>\mathrm{n}_{1}$. With reference to the current time, at any time in the past,

$$
\begin{aligned}
&\left(E_{\text {pot }}\right)_{t} \cong-\left(\frac{M_{t}}{M_{0}}\right)\left(\frac{e^{2}}{4 \pi \varepsilon_{0} G m_{p}^{2}}\right)\left(\frac{e^{2}}{4 \pi \varepsilon_{0} M_{0} c^{2}}\right)\left(\frac{c^{4}}{n^{2} G}\right) \\
&\left(E_{\text {kin }}\right)_{t} \cong \cong \frac{1}{2}\left(\frac{M_{t}}{M_{0}}\right)\left(\frac{e^{2}}{4 \pi \varepsilon_{0} G m_{p}^{2}}\right)\left(\frac{e^{2}}{4 \pi \varepsilon_{0} M_{0} c^{2}}\right)\left(\frac{c^{4}}{n^{2} G}\right) \\
&\left(E_{\text {photon }}\right)_{t} \cong \frac{1}{2}\left(\frac{M_{t}}{M_{0}}\right)\left(\frac{e^{2}}{4 \pi \varepsilon_{0} G m_{p}^{2}}\right)\left(\frac{e^{2}}{4 \pi \varepsilon_{0} M_{0} c^{2}}\right)\left[\left(\frac{c^{4}}{G}\right)\left(\frac{1}{n_{1}^{2}}-\frac{1}{n_{2}^{2}}\right)\right] \\
& \cong \frac{h c}{\lambda_{G}} \\
&\left(E_{\text {pot }}\right)_{t} \propto\left(\frac{e^{2}}{4 \pi \varepsilon_{0} M_{0} c^{2}}\right) \\
&\left(E_{\text {pot }}\right)_{t} \propto\left(\frac{c^{4}}{n^{2} G}\right) \\
&\left(E_{\text {pot }}\right)_{t} \propto\left(\frac{M_{t}}{M_{0}}\right) \\
&\left(E_{\text {pot }}\right)_{t} \propto\left(\frac{e^{2}}{4 \pi \varepsilon_{0} G m_{p}^{2}}\right)
\end{aligned}
$$

In this way observed cosmic redshift can be understood and with reference to the observed $\lambda_{G}$ of the distant galaxy, its corresponding $H_{t}$ can be estimated as follows.

$$
\begin{aligned}
& \frac{H_{t}}{H_{0}} \cong\left\{\frac{1}{2}\left(\frac{e^{2}}{4 \pi \varepsilon_{0} G m_{p}^{2}}\right)\left(\frac{e^{2}}{4 \pi \varepsilon_{0} M_{0} c^{2}}\right)\left[\left(\frac{c^{4}}{G}\right)\left(\frac{1}{n_{1}^{2}}-\frac{1}{n_{2}^{2}}\right)\right]\right\}\left(\frac{h c}{\lambda_{G}}\right)^{-1} \\
& \frac{M_{t}}{M_{0}} \cong\left(\frac{h c}{\lambda_{G}}\right)\left\{\frac{1}{2}\left(\frac{e^{2}}{4 \pi \varepsilon_{0} G m_{p}^{2}}\right)\left(\frac{e^{2}}{4 \pi \varepsilon_{0} M_{0} c^{2}}\right)\left[\left(\frac{c^{4}}{G}\right)\left(\frac{1}{n_{1}^{2}}-\frac{1}{n_{2}^{2}}\right)\right]\right\}^{-1}
\end{aligned}
$$

The current reduced Planck's constant can be fitted as follows.

$$
\hbar_{0} \cong \sqrt{\frac{M_{0}}{m_{e}}} \cdot \frac{G m_{p} m_{e}}{c}
$$

Here $\frac{M_{0}}{m_{e}} \cong \frac{c^{3}}{2 G H_{0} m_{e}} \cong \frac{c}{H_{0}} \div \frac{2 G m_{e}}{c^{2}}$ can be considered as the virtual number of electrons in the current universe. Based on $\left(\frac{c^{4}}{n^{2} G}\right)$, its discrete
form can be expressed as follows.

$$
\begin{aligned}
& n \hbar_{0} \cong \sqrt{\frac{M_{0}}{m_{e}}} \cdot \frac{n G m_{p} m_{e}}{c} \\
& \text { Based on }\left(\frac{c^{4}}{n G}\right), \\
& \sqrt{n} \hbar_{0} \cong \sqrt{\frac{M_{0}}{m_{e}}} \cdot \frac{\sqrt{n} G m_{p} m_{e}}{c}
\end{aligned}
$$

At any time in the past,

$$
\hbar_{t} \cong \sqrt{\frac{m_{e}}{M_{t}}} \cdot \frac{G m_{p} M_{0}}{c}
$$

\section{The Absolute Cosmic Time}

The concept of time has puzzled human beings for centuries. Many physicists have suggested that the problem is that time is not actually real but a property that emerges from something more fundamental. In reality, the problem of determining the age of the universe is closely tied to the problem of determining the values of the cosmological parameters. Calculating the age of the universe is accurate only if the assumptions built into the models being used to estimate it are also accurate. Time seems to be a silent observer in the presently believed 'cosmic inflation'. Assuming a rapid rate of cosmic expansion and steady rate of time flow may not be reasonable. If space-time are interrelated then 'space' and 'time' both should simultaneously follow the momentary rapid exponential expansion. For example if space expands by a factor $10^{78}$ in volume, time should also increase by a factor $10^{26}$ in magnitude. In this regard for estimating the absolute magnitude of the cosmic time, the authors propose the following semi empirical relation.

$$
\begin{aligned}
& t . H_{t} \cong \frac{3 H_{t}^{2} c^{2}}{8 \pi G a T_{t}^{4}} \cong\left[1+\ln \left(\frac{H_{C}}{H_{t}}\right)\right]^{2} \\
& a T_{t}^{4} \cong\left(\frac{c / H_{t}}{c t}\right) \frac{3 H_{t}^{2} c^{2}}{8 \pi G} \cong \frac{3 H_{t} c^{2}}{8 \pi G t}
\end{aligned}
$$

where $t \geq 1 / H_{C}$. It can be expressed in the following way also.

$$
\left(a T_{t}^{4}\right)(t) \cong \frac{3 H_{t} c^{2}}{8 \pi G}
$$

where $t \geq 1 / H_{C}$. This can be considered as one very crucial and absolute application of the assumed cosmic age.

From above assumption or relation (18), current cosmic age can be obtained as follows.

$$
\begin{aligned}
t_{0} & \cong\left[1+\ln \left(\frac{H_{C}}{H_{0}}\right)\right]^{2} \frac{1}{H_{0}} \cong 8.89 \times 10^{21} \text { sec. } \\
& \approx 282 \times 10^{12} \text { years } \cong 282 \text { trillion years. }
\end{aligned}
$$


With this large time - smooth cosmic expansion, cosmic isotropy, super novae dimming and magnetic monopole vanishing etc can be understood. In Indian vedic cosmology, total age of the universe is 311 trillion years $[1,2,47]$. This is a striking and surprising coincidence. It can be suggested that, modern cosmology and Indian vedic cosmology can be studied in a unified manner. This obtained magnitude of current cosmic age plays a very interesting role in fitting the strength of electromagnetic interaction in the following way.

$$
\left(e^{\frac{1}{\alpha}}\right)_{t}^{-2}\left(\frac{H_{C}}{H_{t}}\right)^{2} \cong t H_{t} \cong \frac{3 H_{t}^{2} c^{2}}{8 \pi G a T_{t}^{4}}
$$

\section{Discussion}

In cosmology, a Hubble volume, or Hubble sphere, is a spherical region of the Universe surrounding an observer beyond which objects recede from that observer at a rate greater than the speed of light due to the expansion of the Universe. Objects at the Hubble limit have an average comoving speed of $c$ relative to an observer on the Earth so that, in a universe with constant Hubble parameter, light emitted at the present time by objects outside the Hubble limit would never be seen by an observer on Earth. That is, Hubble limit would coincide with a cosmological event horizon (a boundary separating events visible at some time and those that are never visible). By considering the growing Hubble volume as the volume of a primordial growing black hole, in this paper the authors proposed different applications of the Hubble volume and Hubble mass in cosmology as well as in microscopic physics.

According to N. J. Poplawski, torsion in the ECSK gravity provides a theoretical explanation for a scenario, according to which every black hole produces a new, baby universe inside and becomes an Einstein-Rosen bridge (wormhole) that connects this universe to the parent universe in which the black hole exists. At extremely high densities, much larger than nuclear densities, torsion manifests itself as a force that counters gravitational attraction, preventing matter in a black hole from compressing to a singularity. Instead, matter reaches a state of finite, extremely high density, stops collapsing, undergoes a bounce, and starts rapidly expanding as a new universe. Extremely strong gravitational fields near the bounce cause an intense particle production, increasing the mass inside a black hole by many orders of magnitude. Accordingly, our own Universe could be the interior of a black hole existing in another universe.

According to Tianxi Zhang, our entire universe is one massive black hole, within which everything we "see" exists. Over time, as our universe evolves, the black holes that we observe will continue to grow and merge; eventually, all matter in our universe will merge together into one massive singularity. At this time, a new universe would be born within it. Thus in a modern cosmological approach it is ideal to say that the universe may have been borne inside a black hole, and the black holes in our own cosmos might be birthing new universes of their own.

\section{Conclusion}

With vigorous advanced mathematics some of the cosmologists are able to show that observed universe is a black hole. Authors are working on the assumed Hubble volume and Hubble mass in different directions with different applications [48-51] that connect micro physics and macro physics. With the proposed applications it is very clear to say that, without a combined and unified study of cosmology and microscopic physics, one should not deny the concepts of black hole cosmology. Authors repeat the statement that - compared to the
Big bang model, advantage of Black hole cosmology lies in confirming its validity through the ground based atomic and nuclear experimental results.

Even though Quantum mechanics and General theory of relativity both are having independent existence, strong mathematical back ground and good physical beauty, combining them is beyond the scope of current physics standards and demands sound knowledge on unknown and hidden things of atom and the universe. Even though 'dark energy' holds $70 \%$ of the unseen matter content of the universe, its role in understanding the basic concepts of unification is very insignificant. Even though SUSY is having excellent theoretical support and in-depth mathematical back ground, based on SUSY concepts so far no single SUSY boson could be detected in the Large Hadron Collider. This puzzling issue casts doubt on the continued existence of SUSY. In a nutshell, it is very clear that something is missing from our 'unification' knowledge net! Missing knowledge can be obtained only through intellectual thinking, mathematical modeling, probing the atomic nucleus and universe to the possible extent, constructing semi empirical relations among physical constants of various interdisciplinary branches of physics with all possible interpretations and so on. Which way/method is the best - will be decided by future experiments, observations and interpretations. As it is interconnected with all branches of physics, 'semi empirical approach' seems be the easiest and shortcut way. It sharpens and guides human thinking ability in understanding the reality of unification. For any theoretical concept or mathematical model or semi empirical relation, 'workability' is more important than its inner beauty and 'workability' is the base of any semi empirical approach.

Understanding and connecting 'tiny atom' and the 'gigantic universe' is really a very big challenging task. Bringing 5 different branches of basic physics into 'Single frame' is a very tough job. The basic idea of unification is -1) To minimize the number of physical constants and to merge a group of different fundamental constants into one compound physical constant with appropriate unified interpretation and 2) To merge and minimize various branches of physics. In this journey, the first step is to see the numerical coincidences, second step is to interpret the numerical coincidences and the third step is to synchronize the current interpretations and new interpretations. When the new interpretation disagrees with the current interpretation, generally with the help of emerging science and technology, discrepancies can be resolved with future observations, experiments and analysis.

Based on the current observations and obtained null results of $\frac{d}{d t}\left(T_{t}\right), \frac{d}{d t}\left(H_{t}\right)$ and $\frac{d}{d t}(-)$ it can be suggested that cosmic acceleration and dark energy are pure mathematical concepts and there exist no physical base behind their confirmation. Now the key leftover things are nucleosynthesis and structure formation. Authors are working in this direction. As nuclear binding energy was zero at the beginning of cosmic evolution, by considering the time dependent variable nature of magnitudes of the semi empirical mass formula energy coefficients [52] it is possible to show that, at the beginning of formation of nucleons, nuclear stability is maximum for light atoms only. If so it can be suggested that, from the beginning of formation of nucleons, in any galaxy, maximum scope is being possible only for the survival of light atoms and this may be the reason for the accumulation and abundance of light atoms in large proportion.

\section{References}

1. Seshavatharam UVS (2010) Physics of rotating and expanding black hole universe. Progress in Physics 2: 7-14 
Citation: Seshavatharam UVS, Lakshminarayana S (2014) Black Hole Cosmology: A Biological Boom. Astrobiol Outreach 2:108. doi: 10.4172/23322519.1000108

Page 10 of 10

2. Seshavatharam UVS (2012) The Primordial Cosmic Black Hole and the Cosmic Axis of Evil. International Journal of Astronomy 1: 20-37.

3. Seshavatharam UVS (2013) Light speed rotating black holes: The special holes. International Journal of Advanced Astronomy 1: 13-20.

4. Seshavatharam UVS, Lakshminarayana S (2013) Microscopic Physical Phenomena in Black Hole Cosmos Rotating at Light Speed. Prespacetime Journal 4: 884-922

5. Zhang TX, Frederic C (2013) Acceleration of black hole universe. Astrophysics and Space Science349: 567-573.

6. ZhangT (2010) Cosmic microwave background radiation of black hole universe Astrophysics and Space Science 330: 157-165.

7. ZhangT (2012) Quasar Formation and Energy Emission in Black Hole Universe Progress in Physics 3: 48-53.

8. Poplawski NJ (2010) Radial motion into an Einstein-Rosen bridge. Physics Letters B 687: 110-113.

9. Poplawski NJ (2012) Big bounce from spin and torsion. General Relativity and Gravitation 44: 1007-1014.

10. Poplawski NJ Energy and momentum of the Universe.

11. PoplawskiNJ The universe as a black hole in isotropic coordinates.

12. Pourhasan R, Afshordi N, Mann RB (2013) Did a hyper black hole spawn the universe? Nature - International weekly journal of science. 13 September 2013 , doi:10.1038/nature.2013.13743, arXiv:1309.1487v2.

13. Hawking SW (1988) A Brief History of Time. Bantam Dell Publishing Group

14. Hawking SW, Ellis GFR (1973) The Large-Scale Structure of SpaceTime. Cambridge University Press. ISBN 0-521-20016-4.

15. Hubble EP (1929) A relation between distance and radial velocity among extragalactic nebulae 15: 168-173.

16. Hubble EP (1947) The 200-inch telescope and some problems it may solve PASP, 59: 153-167

17. Pathria RK (1972) The Universe as a Black Hole. Nature 240:298-299.

18. Good IJ (1972) Chinese universes. Physics Today 25: 15

19. Joel S, Blake T (2003) Shock-wave cosmology inside a black hole. ProcNatlAcadSci U S A.

20. Andy G, Joseph PC (2013) Cosmological natural selection and the purpose of the universe. Complexity 18: 48-56.

21. Chul-Moon Yoo et al. Black Hole Universe. Time evolution. Phys. Rev. Lett. 111, 161102(2013)

22. Michael J. Longo, Detection of a Dipole in the Handedness of Spiral Galaxies with Redshifts z 0.04, Phys. Lett. B 699, 224-229 2011.

23. S.-C. Su and M.-C. Chu. Is the universe rotating? Astrophysical Journal, 703 354. 2009

24. J. D. McEwen, et al. Bayesian analysis of anisotropic cosmologies: Bianchi VIlh and WMAP.Mon. Not. R. Astron. Soc. 000, 1-15 (2013). arXiv:1303.3409v1.

25. Chechin LM (2013) On the Modern Status of the Universe Rotation Problem. Journal of Modern Physics 4: 126-132.

26. SivaramC, KenathA (2012) Primordial Rotation of the Universe, Hydrodynamics Vortices and Angular Momenta of Celestial Objects. The Open Astronomy Journal 5: 7-11.

27. Sidharth BG (2010) Is the Universe Rotating? Prespacetime Journal. 1: 11681173

28. Marcelo SB, Fernando G (2013) Local and Global Stability of the Universe Journal of Modern Physics 4: 7-9.

29. Robert V Gentry. New Cosmic Center Universe Model Matches Eight of Big Bang's Major Predictions Without The F-L Paradigm. CERN preprint, EXT2003-022, 14 Apr 2003

30. ChaplineG (2006) Tommy Gold Revisited: Why Does Not The Universe Rotate? AIP Conf.Proc 822:160-165.

31. Dmitri R (2009) On the Speed of Rotation of Isotropic Space: Insight into the Redshift Problem. The Abraham Zelmanov Journal 2: 208-223.
32. Kurt G (1950) Rotating Universes in General Relativity Theory. Proceedings of the international Congress of Mathematicians in Cambridge 1: 175-81

33. Hawking SW (1969) On the rotation of the universe. Mon. Not. Royal. Astr. Soc 142: $129-141$

34. Novello M, Reboucas MJ (1979) Rotating universe with successive causal and noncausal regions. Phys. Rev. D 19: 2850-2852.

35. Barrow JD, Juszkiewicz R, Sonoda DH (1985) Universal rotation - How large can it be? Mon Not R AstronSoc 213: 917-943.

36. Surdin M (1995) The Rotating Universe. Physics Essays 8: 282-284.

37. Christopher S (2013) Reynolds. Astrophysics: Black holes in a spin. Nature 494: 432-433.

38. Louis M (1988) A New Non-Doppler Redshift. Physics Essays 1:24-32.

39. Louis Marmet On the Interpretation of Red-Shifts: A Quantitative Comparison of Red-Shift Mechanisms.

40. Bennett CL, Larson D,Weiland JL, JarosikN,Hinshaw G, et al. (2012) NineYear Wilkinson Microwave Anisotropy Probe (WMAP) Observations: Fina Maps and Results.

41. J. Huchara. Estimates of the Hubble Constant, 2010. Harvard-Smithsonian Center for Astrophysics

42. Freedman WL, Madore BF, Gibson BK, Ferrarese L, Kelson DD, et al. (2001) Final Results from the Hubble Space Telescope Key Project to Measure the Hubble Constant. The Astrophysical Journal 553: 47-72.

43. Beringer J. Particle Data Group. Phys. Rev. D86, 010001 (2012)

44. Seshavatharam UVS, Lakshminarayana S (2012) The Reduced Planck's Constant, Mach's Principle, Cosmic Acceleration and the Black Hole Universe. Journal of Physical Science and Application 2: 441-447.

45. Lianxi M, Jiacai N, Junjun Y (2009) Two forms of Wien's displacement law. Lat Am. J. Phys. Educ 3:1870-9095.

46. Hawking SW (1975) Particle creation by black holes. Commun Math Phys43 199-220.

47. Ebenezer B (1860) Translation of the Surya-Siddhanta. A text-book of Hindu Astronomy. Journal of the American Oriental Society6: 141-498.

48. Seshavatharam, UVS, Lakshminarayana S (2013) Applications of Hubble Volume in Atomic Physics, Nuclear Physics, Particle Physics, Quantum Physics and Cosmic Physics. Journal of Nuclear Physics, Material Sciences, Radiation and Applications 1: 45-60.

49. Seshavatharam UVS, Lakshminarayana S (2013) To confirm the existence of Black hole cosmology. International Journal of Advanced Astronomy 2: 21-36.

50. Seshavatharam UVS, Lakshminarayana S (2012) Hubble Volume and the Fundamental Interactions, International Journal of Astronomy1: 87-100

51. Seshavatharam UVS, Lakshminarayana S, Sai BVST (2013) Is red shift an index of galactic'atomic light emission' mechanism? International Journal of Physics1: 49-64.

52. Seshavatharam UVS, Lakshminarayana S (2013) New Concepts \& SemiEmpirical Fittings in Understanding SUSY \& the Four Cosmological Interactions. Prespacetime Journal4: 1027-1038. 